\title{
On Non-Commutative Integrable Burgers Equations
}

Metin Gürses, Atalay Karasu, Refik Turhan

To cite this article: Metin Gürses, Atalay Karasu, Refik Turhan (2010) On Non-

Commutative Integrable Burgers Equations, Journal of Nonlinear Mathematical Physics 17:1, 1-6, DOI: https://doi.org/10.1142/S1402925110000532

To link to this article: https://doi.org/10.1142/S1402925110000532

Published online: 04 January 2021 
(C) M. Gürses, A. Karasu and R. Turhan

DOI: $10.1142 / \mathrm{S} 1402925110000532$

\title{
ON NON-COMMUTATIVE INTEGRABLE BURGERS EQUATIONS
}

\author{
METIN GÜRSES \\ Department of Mathematics, Faculty of Sciences \\ Bilkent University, 06800 Ankara, Turkey \\ ATALAY KARASU \\ Department of Physics, Faculty of Arts and Sciences \\ Middle East Technical University, 06531 Ankara, Turkey \\ REFIK TURHAN \\ Department of Engineering Physics \\ Ankara University, 06500 Ankara, Turkey \\ Received 1 April 2009 \\ Accepted 16 September 2009
}

\begin{abstract}
We construct the recursion operators for the non-commutative Burgers equations using their Lax operators. We investigate the existence of any integrable mixed version of left- and right-handed Burgers equations on higher symmetry grounds.
\end{abstract}

Keywords: Integrability; Burgers equation; non-commutativity; symmetries; recursion operators.

\section{Introduction}

Non-commutative generalizations of the classical nonlinear evolution equations in $(1+1)$ dimensions were classified according to the symmetry based integrability in [1]. In this classification, (besides some multi-component equations) one-component non-commutative versions of a Korteweg-de Vries (ncKdV), a Potential KdV (ncPKdV), two Modified KdV (ncMKdV1, ncMKdV2) and two (left- and right-handed) nc-Burgers equations are observed to have higher symmetry in a certain weighting scheme of symmetries. Recursion operators for ncKdV, ncPKdV and ncMKdV1 were given in [2] and the one for ncMKdV2 in [3]. In [2], classification [1] is shown to be complete under the assumed weighting scheme of equations.

Possession of a higher symmetry is a necessary condition which needs to be supplemented by either a recursion operator or a master symmetry for an equation to be integrable in the symmetry sense $[4,5]$. A recursion operator generates infinite hierarchy of symmetries by mapping a symmetry to another endlessly. Master symmetries do the same as adjoint action. Therefore ncKdV, ncPKdV, ncMKdV1 and ncMKdV2 are integrable equations. 
As for the nc-Burgers equations, their integrability is proven by a master symmetry [2]. Moreover, they are shown to be linearizable by a non-commutative version of the Cole-Hopf transformation. Their symmetry hierarchies are obtainable from the "higher heat equations" and they admit auto-Bäcklund transformations [6,7]. Some exact solutions of the associated linearized (nc-Heat) equation were obtained in $[8,9]$. So, nc-Burgers equations are one of the best studied equations among the known non-commutative integrable equations. There is one missing point however; currently no recursion operator of nc-Burgers equations is known. It is even claimed that there exists none [2].

Integrability has many aspects. A symmetry integrable equation may further possess a hierarchy of conservation laws, or a Lax formulation, auto-Bäcklund transformations, Hirota bilinear formulation, Painlave property etc. In general, there is not a well established correspondence among these structures. But demonstration of one of these structures is regarded as a strong indication (and a good motivation to search) for other structures of an equation.

Recently, a new nc-Burgers equation, which is a particular parametric mixture of the left- and right-handed nc-Burgers equations, was introduced with a Lax pair in [9]. Despite having a Lax formulation, absence of this mixed nc-Burgers equation in the former symmetry and the relevant structure studies is remarkable.

In this paper, starting from a Kupershmidt type hierarchy of Lax representations, we construct both the time independent and the time dependent recursion operators of the ncBurgers equations by the method introduced in [3]. Our construction leads to only recursion operators of the left- or right-handed nc-Burgers equations. The Lax representation given for the mixed version of nc-Burgers equation does not lead to a recursion operator of the equation by the mentioned technique. Therefore, we reinvestigate the possibility of having an integrable mixed version of nc-Burgers equation on higher symmetry grounds again. This time, however, we do the symmetry analysis by relaxing the weighting constraints taken in $[1,2]$. We comment on sufficiency of having a Lax pair to be integrable in the symmetry sense.

\section{Construction of Recursion Operators}

From here on, by nc-Burgers equation we shall specifically refer to the right-handed ncBurgers equation

$$
u_{t}=u_{2 x}+2 u u_{x}
$$

and present the explicit results pertaining to this version only. This is because all the results given here correspond to that of left-handed nc-Burgers equation by an interchange of left multiplication operator $L_{\psi}(\phi)=\psi \phi$ with the right multiplication $R_{\psi}(\phi)=\phi \psi$ at their every occurrence.

The Lax representation for the nc-Burgers hierarchy with

$$
\mathcal{L}=D_{x}+L_{u}
$$

is given by

$$
\mathcal{L}_{t_{n}}=\left[\mathcal{A}_{n}, \mathcal{L}\right]
$$

where $\mathcal{A}_{n}=\left(\mathcal{L}^{n}\right)_{\geq 1}$ and $D_{x}$ denotes the total derivative with respect to $x$. 
When a hierarchy of Lax pairs is known, a technique for constructing recursion operator for the associated symmetry hierarchy is given in [3]. This technique is based mainly on the identification of the relation between the Lax representations of the individual symmetries in the hierarchy. For the detailed explanation of the method and explicit examples we refer to $[3]$.

The relation among the Lax representations of the individual equations (symmetries) in the nc-Burgers hierarchy is

$$
\mathcal{L}_{t_{n+1}}=\mathcal{L} \mathcal{L}_{t_{n}}+\left[\mathcal{T}_{n}, \mathcal{L}\right]
$$

With the ansatz for the remainder $\mathcal{T}_{n}=a_{n} D_{x}+b_{n}$, solution of this operator equality gives $a_{n}=a d_{\mathcal{L}}^{-1} u_{n}$ and arbitrary $b_{n}$. Therefore we have the following recursion formula

$$
u_{n+1}=\left(D_{x}+L_{u}\right) u_{n}+R_{u_{x}} a d_{\mathcal{L}}^{-1} u_{n}-a d_{\mathcal{L}} b_{n}
$$

among the successive symmetries. Here $a d_{\mathcal{L}}=D_{x}+L_{u}-R_{u}$.

Considering the vector space structure of symmetries on which the recursion operators act, we first choose $b_{n}=0$ and arrive at the time independent recursion operator of the nc-Burgers equation which is

$$
\mathcal{R}_{1}=D_{x}+L_{u}+R_{u_{x}} a d_{\mathcal{L}}^{-1}
$$

To show that $\mathcal{R}_{1}$ is a conventional recursion operator for the nc-Burgers hierarchy, one needs to prove that it satisfies the basic defining condition [4]

$$
\mathcal{R}_{t}=[\mathcal{F}, \mathcal{R}]
$$

where $\mathcal{F}=D_{x}^{2}+2 L_{u} D_{x}+2 R_{u_{x}}$ is the Frechet derivative of the right-hand side of nc-Burgers equation (1). In the present case however, it is more convenient to verify this basic condition in its equivalent form [10]

$$
\mathcal{M}_{t}-\mathcal{F} \mathcal{M}=\mathcal{M N}^{-1}\left(\mathcal{N}_{t}-\mathcal{F N}\right)
$$

where $\mathcal{R}_{1}=\mathcal{M N}^{-1}$ with

$$
\mathcal{M}=\left(D_{x}+L_{u}\right) a d_{\mathcal{L}}+R_{u_{x}}, \quad \mathcal{N}=a d_{\mathcal{L}}
$$

It can be straightforwardly verified that Eq. (8) indeed holds and therefore $\mathcal{R}_{1}$ is a recursion operator for nc-Burgers equation. It generates an infinite hierarchy of symmetries if an initial one is given. The first few symmetries starting from $\sigma_{0}=u_{x}$ are

$$
\begin{aligned}
& \sigma_{1}=u_{2 x}+2 u u_{x}, \\
& \sigma_{2}=u_{3 x}+3 u u_{2 x}+3 u_{x}{ }^{2}+3 u^{2} u_{x}, \\
& \sigma_{3}=u_{4 x}+4 u u_{3 x}+4 u_{x} u u_{x}+4 u_{2 x} u_{x}+6 u^{2} u_{2 x}+4 u^{3} u_{x}+6 u_{x} u_{2 x}+8 u u_{x}{ }^{2} .
\end{aligned}
$$

As in the commutative Burgers equation, there is another recursion operator of the ncBurgers equation which is explicitly time dependent. We can determine the time-dependent 
recursion operator $\mathcal{R}_{2}$ with the help of the $b_{n}$ and by inspection. Hence choosing $b_{n}=$ $\left(t D_{x}+t L_{u}+\frac{x}{2}\right) a d_{\mathcal{L}}^{-1} u_{n}$ in (5) we get the time-dependent recursion operator

$$
\mathcal{R}_{2}=a d_{\mathcal{L}}\left(t D_{x}+t L_{u}+\frac{x}{2}\right) a d_{\mathcal{L}}^{-1}
$$

Again, as in the commutative case, this time-dependent recursion operator $\mathcal{R}_{2}$ is a weak recursion operator meaning that despite satisfying the basic condition (8), this recursion operator fails to generate higher symmetries correctly. Going through the algorithm $[11,12]$, the corrected time-dependent recursion operator for the nc-Burgers equation is obtained to be

$$
\mathcal{R}_{2}=a d_{\mathcal{L}}\left(t D_{x}+t L_{u}+\frac{x}{2}\right) a d_{\mathcal{L}}^{-1}+\frac{1}{2}\left(\frac{1}{2}+t L_{u_{x}}\right) D_{t}^{-1} \Pi a d_{\mathcal{L}}
$$

where $\Pi$ is the projection operator defined as $\Pi h\left(t, x, u, u_{x}, \ldots\right)=h(t, 0,0, \ldots)$ for any function $h$. The first few symmetries are

$$
\begin{aligned}
\sigma_{0}= & \frac{1}{2}+t u_{x} \\
\sigma_{1}= & t^{2}\left(u_{2 x}+2 u u_{x}\right)+t\left(u+x u_{x}\right)+\frac{1}{2} x \\
\sigma_{2}= & t^{3}\left(u_{3 x}+3 u u_{2 x}+3 u_{x}^{2}+3 u^{2} u_{x}\right)+t^{2}\left(3 u_{x}+\frac{3}{2} x u_{2 x}+3 x u u_{x}+\frac{3}{2} u^{2}\right) \\
& +t\left(\frac{3}{4}+\frac{3}{4} x^{2} u_{x}+\frac{3}{2} u x\right)+\frac{3}{8} x^{2} .
\end{aligned}
$$

\section{Mixed nc-Burgers Equations}

The mixed nc-Burgers equation having an arbitrary constant $\alpha$

$$
v_{t}=v_{2 x}+(\alpha-2) v_{x} v+\alpha v v_{x}
$$

was introduced with the Lax pair

$$
\mathcal{L}_{\text {mixed }}=D_{x}+v, \quad \mathcal{A}_{\text {mixed }}=D_{x}^{2}+2 v D_{x}+2 v_{x}+\alpha v^{2}
$$

in [9]. Even though the mixed nc-Burgers equation (13) admits the particular Lax formulation (14) which may be regarded as indicating integrability. This equation has not shown up in symmetry classifications $[1,2]$. Moreover, we have already obtained the recursion operators that $\mathcal{L}_{\text {mixed }}$ can give. They are only the recursion operators of the nc-Burgers equation (1) admitting Lax representations $\mathcal{L}=D_{x}+L_{u}$ or $\mathcal{L}=D_{x}+R_{u}$.

Therefore, here we reinvestigate a slightly generalized mixed nc-Burgers equations for higher symmetry. In our specific attempt, we relax the weighting scheme used in $[1,2]$ for the selection of the terms to be included in the candidate symmetry. We included all the terms with polynomial and derivative orders up to four. As a result, we have the following proposition. 
Proposition. The equation of form

$$
u_{t}=u_{2 x}+a u u_{x}+b u_{x} u
$$

with $a, b \in \mathbb{R}$ and $a b \neq 0, u$ is non-commutative,

(i) does not admit any higher symmetry from the class of equations

$$
\begin{aligned}
u_{t}= & \nu(t, x)+\sum_{i=0}^{4} \alpha^{i}(t, x) u_{i x}+\sum_{i, j=0}^{4} \beta^{i j}(t, x) u_{i x} u_{j x}+\sum_{i, j, k=0}^{4} \gamma^{i j k}(t, x) u_{i x} u_{j x} u_{k x} \\
& +\sum_{i, j, k, l=0}^{4} \delta^{i j k l}(t, x) u_{i x} u_{j x} u_{k x} u_{l x},
\end{aligned}
$$

(ii) admits only the Lie-point symmetries

$$
\begin{aligned}
\sigma_{1} & =u_{t}, \\
\sigma_{2} & =u_{x}, \\
\sigma_{3} & =2 t u_{t}+x u_{x}+u, \\
\sigma_{4} & =1+(a+b) t u_{x},
\end{aligned}
$$

(iii) in particular, when $b=-a, \sigma_{1}, \sigma_{2}, \sigma_{3}$ remains as they are but $\sigma_{4}=1$ generalizes to $\sigma_{4}=h(t, x)$ where $h(t, x)$ is a solution of $h_{t}=h_{x x}$.

So, to the extent of the above proposition, integrable versions of nc-Burgers equations are only the left- and right-handed ones which are already given in [1]. We claim that there does not exist either a Lax hierarchy or a recursion operator for the mixed nc-Burgers equation (13).

Nevertheless, a non-integrable equation with a Lax formulation is not unusual. Such (commutative) equations are shown to exist in [13] and further investigated in $[14,15]$.

One of the authors A.K. is thankful to Sergei Sakovich for various valuable discussions. This work is partially supported by the Scientific and Technological Research Council of Turkey (TUBITAK) and Turkish Academy of Sciences (TUBA).

\section{References}

[1] P. J. Olver and V. V. Sokolov, Commun. Math. Phys. 193 (1998) 245.

[2] P. J. Olver and J. P. Wang, Proc. London. Math. Soc. 81 (2000) 566.

[3] M. Gürses, A. Karasu and V. V. Sokolov, J. Math. Phys. 40 (1999) 6473.

[4] P. J. Olver, Applications of Lie Groups to Differential Equations, Second Edition, Graduate Texts in Mathematics, Vol. 107 (Springer-Verlag, New York, 1993).

[5] M. Blaszak, Multi-Hamiltonian Theory of Dynamical Systems (Springer, Berlin, 1998).

[6] B. A. Kupershmidt, KP or mKp: Noncommutative Mathematics of Lagrangian, Hamiltonian, and Integrable Systems (American Mathematical Society, Providence, 2000).

[7] B. A. Kupershmidt, J. Nonlin. Math. Phys. 12 (2005) 539.

[8] L. Martina L and O. K. Pashaev, Burgers equation in non-commutative space-time, Preprint hep-th/0302055.

[9] M. Hamanaka and K. Toda, J. Phys. A 36 (2003) 11981.

[10] A. Karasu (Kalkanli), A. Karasu and S. Yu. Sakovich, Acta Appl. Math. 83 (2004) 85.

[11] J. A. Sanders and J. P. Wang, Physica D. 149 (2001) 1. 
[12] M. Gürses, A. Karasu and R. Turhan, J. Nonlin. Math. Phys. 9 (2002) 210.

[13] F. Calogero and M. C. Nucci, J. Math. Phys. 32 (1991) 72.

[14] S. Yu. Sakovich, Acta Appl. Math. 83 (2004) 69.

[15] J. P. Wang, J. Phys. A: Math. Theor. 42 (2009) 362004. 